$\alpha_{3, n}=4+3 i, \alpha_{4, n}=-2 i$, and the corresponding equation has the roots $z_{1}, z_{2}, z_{3}, z_{4}$. This occurs because of the presence of the double root $z_{2}=2$. For the same reason, all fifth order systems will be dependent.

A practical difficulty may arise with fixed decimal point machinery in that $s_{n}$ may go off scale either on the right or the left. This will happen for sufficiently large $n$ when $\left|z_{1}\right| \neq 1$. This difficulty may be overcome by a transformation $z^{\prime}=z / r$, where $r=$ $\left|\alpha_{M, n}\right|^{1 / M}, M$ being the order of a convergent process, $n$ being "sufficiently" large. In our example we have $r=\left|\alpha_{2, n}\right|^{1 / 2}$. For $n$ as small as three, $\left|s_{n}\right|$ would remain in a very satisfactory region.

\title{
AERODYNAMIC FORCES ON A SLOTTED FLAT PLATE*
}

BY EDMUND PINNEY (University of California, Berkeley)

The derivation of the flat plate approximation to the aerodynamic forces exerted by an incompressible fluid on an airfoil is simple and is to be found in most textbooks. The present note treats the less simple case in which the airfoil contains slots oriented parallel to its length. This problem is of importance in the theory of suspension bridges where slotted roadbeds are used to cut down aerodynamically driven oscillations.

The spaces between the slots will be called "lanes". Let $V$ be the velocity of flow, and $\alpha$ the angle of attack, assumed to be small. Let the plate occupy the interval $-b \leq$ $x \leq b, y=0$ of a rectangular coordinate system, and let $u(z), v(z)$ be the velocity components of the fluid parallel to the $x$ and $y$ axes, respectively, at the point $z=x+i y$. Then, if powers of $\alpha$ higher than the first are neglected,

$$
u(z) \rightarrow V, \quad v(z) \rightarrow V \alpha \quad \text { as }|z| \rightarrow \infty .
$$

By two-dimensional potential theory, $v(z)+i u(z)$ is an analytic function of $z$ away from the plate. Consider

$$
v(z)+i u(z)=i V+V \alpha \prod_{1}^{N}\left(z-t_{n} b\right)^{1 / 2}\left(z-l_{n} b\right)^{-1 / 2}
$$

where $x=t_{n} b$ and $x=l_{n} b$ are the trailing and leading edges, respectively, of the $n$-th lane, and $N$ is the total number of lanes. The lanes are numbered from left to right so that

$$
l_{1}=-1, \quad t_{n+1}>t_{n}, \quad l_{n+1}>l_{n}, \quad l_{n+1}>t_{n}, \quad t_{N}=1 .
$$

As $z \rightarrow \infty$, it is seen that (1) is satisfied.

In the $n$-th lane $l_{n} b<x<t_{n} b$, and by (2),

$$
v(x \pm i 0)+i u(x \pm i 0)=i V \pm i V \alpha \prod_{1}^{N}\left|x-t_{n} b\right|^{1 / 2}\left|x-l_{n} b\right|^{-1 / 2} .
$$

Therefore

$$
u(x \pm i 0)=V \pm V \alpha \prod_{1}^{N}\left|x-t_{n} b\right|^{1 / 2}\left|x-l_{n} b\right|^{-1 / 2}, \quad v(x \pm i 0)=0 .
$$

*Received Sept. 22, 1947. 
Therefore the velocity distribution of (2) satisfies the boundary conditions on the lanes. Also the velocity is finite when $z=t_{n} b$, so the Kutta condition is satisfied on the trailing edge of each lane.

By Bernoulli's theorem,

$$
p+\frac{1}{2} \rho\left(u^{2}+v^{2}\right)=\text { const. }
$$

where $p$ is the pressure and $\rho$ is the density of the air. The quantities $p$ and $u$ are discontinuous on the lanes. Let $p_{+}, u_{+}$be their values on the upper side, and $p_{-}, u_{-}$their values on the lower side. Then

$$
p_{+}-p_{-}+\frac{1}{2} \rho\left(u_{+}^{2}-u_{-}^{2}\right)=0 .
$$

By (4), on the lanes,

$$
p_{+}-p_{-}+2 \rho V^{2} \alpha \prod_{1}^{N}\left|x-t_{n} b\right|^{1 / 2}\left|x-l_{n} b\right|^{-1 / 2}=0 .
$$

On the slots between the lanes, $p_{+}-p_{-}=0$, of course.

Let

$$
\begin{aligned}
& \Phi(z)=\prod_{1}^{N}\left(z-t_{n} b\right)^{1 / 2}\left(z-l_{n} b\right)^{-1 / 2} /(z-b), \\
& \phi(x)=-\prod_{1}^{N}\left|x-t_{n} b\right|^{1 / 2}\left|x-l_{n} b\right|^{-1 / 2} /(b-x), \quad \text { for } \quad x \text { on a lane, } \\
& =0, \quad \text { for } \quad x \text { on a slot.* }
\end{aligned}
$$

Then, anywhere on the roadbed,

$$
p_{+}-p_{-}=2 \rho V^{2} \alpha(b-x) \phi(x) .
$$

We may now calculate the aerodynamic lift $L$ and moment $M$ from

$$
L=\int_{-b}^{b}\left(p_{+}-p_{-}\right) d x, \quad M=\int_{-b}^{b}\left(p_{+}-p_{-}\right) x d x .
$$

It is possible to obtain these and higher aerodynamic moments, if desired, by evaluating

$$
\int_{-b}^{b}\left[\left(p_{+}-p_{-}\right) /(1-r x / b)\right] d x
$$

and expanding the result in powers of $r$.

If $0<r<1$, by (8),

$$
\frac{b}{r} \int_{-b}^{b} \frac{p_{+}-p_{-}}{x-b / r} d x=2 \pi \rho V^{2} b \alpha\left\{\left(1-\frac{1}{r}\right) \frac{b}{\pi r} \int_{-b}^{b} \phi(x) \frac{d x}{x-b / r}-\frac{1}{\pi r} \int_{-b}^{b} \phi(x) d x\right\} .
$$

Now $\dagger$

$$
\frac{b}{\pi r} \int_{-b}^{b} \phi(x) \frac{d x}{x-b / r}=\frac{b}{r} \Phi\left(\frac{b}{r}\right)
$$

*Pinney, E., Annals of Math. 47, 221-232 (1946). See $\S 3$.

†Pinney, E., loc. cit., formulas (1.1), (3.3). 
Allowing $r$ to approach zero, from $(6),(1 / \pi) \int_{-b}^{b} \phi(x) d x=-1$, so

$$
\int_{-b}^{b}\left(p_{+}-p_{-}\right) \frac{d x}{1-r x / b}=-2 \pi \rho V^{2} b \alpha \frac{1}{r}\left\{1-(1-r) \frac{b}{r} \Phi\left(\frac{b}{r}\right)\right\} .
$$

Therefore, from (6),

$$
\int_{-b}^{b}\left(p_{+}-p_{-}\right) \frac{d x}{1-r x / b}=-2 \pi \rho V^{2} b \alpha \frac{1}{r}\left\{1-\prod_{1}^{N}\left(\frac{1-r t_{n}}{1-r l_{n}}\right)^{1 / 2}\right\} .
$$

Now let

$$
T_{1}=\sum_{1}^{N} t_{n}, \quad T_{2}=\sum_{1}^{N} t_{n}^{2}, \quad L_{1}=\sum_{1}^{N} l_{n}, \quad L_{2}=\sum_{1}^{N} l_{n}^{2} .
$$

Then

$$
\prod_{1}^{N}\left(1-r t_{n}\right) /\left(1-r l_{n}\right)=1-\dot{r}\left(T_{1}-L_{1}\right)+\frac{1}{2} r^{2}\left[\left(T_{1}-L_{1}\right)^{2}-\left(T_{2}-L_{2}\right)\right]+0\left(r^{3}\right),
$$

so

$$
\begin{aligned}
\prod_{1}^{N}\left(1-r t_{n}\right)^{1 / 2}\left(1-r l_{n}\right)^{-1 / 2}=1-\frac{1}{2} r\left(T_{1}-\right. & \left.L_{1}\right) \\
& +\frac{1}{8} r^{2}\left[\left(T_{1}-L_{1}\right)^{2}-2\left(T_{2}-L_{2}\right)\right]+0\left(r^{3}\right) .
\end{aligned}
$$

This may be inserted into (9), expanding the right side in powers of $r$. Expanding the left side in powers of $r$ and equating coefficients, we have

$$
\begin{gathered}
L=-\pi \rho V^{2} b\left(T_{1}-L_{1}\right) \alpha \\
M=\frac{1}{4} \pi \rho V^{2} b^{2}\left[\left(T_{1}-L_{1}\right)^{2}-2\left(T_{2}-L_{2}\right)\right] \alpha,
\end{gathered}
$$

for the aerodynamic lift and moment respectively.

Suppose the slots are symmetrically arranged about the centerline, and suppose $\epsilon$ is the fraction of the total roadbed width occupied by lanes. Then $T_{2}=L_{2}, T_{1}-L_{1}=$ $2 \epsilon$, so

$$
L=-2 \pi \rho V^{2} b \epsilon \alpha, \quad M=\pi \rho V^{2} b^{2} \epsilon^{2} \alpha .
$$

This proves the following theorem.

Theorem. A flat plate airfoil slotted symmetrically about its centerline has the same steady-state aerodynamic lift and moment as an unslotted flat plate of chord equal to the total chord of the slotted plate minus the total width of the slots. 